\title{
Cisalhamento na Linha de Cola de Compensados de Eucalyptus sp. e Adesivo PVA
}

\author{
Bruno Santos Ferreira ${ }^{1}$, Cristiane Inácio de Campos ${ }^{1}$, \\ Marcellus Silveira da Silva ${ }^{1}$, Ivaldo de Domênico Valarelli ${ }^{2}$ \\ ${ }^{1}$ Universidade Estadual Paulista "Júlio de Mesquita Filho" - UNESP \\ ${ }^{2}$ Departamento de Engenharia Mecânica, Universidade Estadual Paulista "Júlio de Mesquita Filho" - UNESP
}

\begin{abstract}
RESUMO
Estudos sobre novos adesivos ou resinas para colagem de madeira e derivados estão sendo realizados com a intenção de melhorar suas propriedades, levando em consideração um menor impacto ambiental. Por este motivo, novas formulações de adesivos de acetato de polivinila (PVA) vêm sendo desenvolvidas, pois estes não possuem em sua composição compostos químicos extremamente poluentes e prejudiciais à saúde, como é o caso de resinas à base de formaldeído, que, por sua vez, são as mais comumente utilizadas hoje em dia para a produção de painéis de madeira. Este trabalho testou três formulações diferentes de adesivos PVA, com duas combinações de tempo e temperatura de prensagem, para a produção de painéis compensados de Eucalyptus sp., obtendo bons resultados com relação à resistência ao cisalhamento na linha de cola, a qual foi superior para os adesivos PVA se comparados com painéis produzidos com ureia e fenol-formaldeído, encontrados na literatura.
\end{abstract}

Palavras-chave: propriedades mecânicas, eucalipto, acetato de polivinila.

\section{Shear Strength at the Bondline of Eucalyptus sp. and PVA Adhesive Plywood}

\begin{abstract}
Studies on new adhesives and resins for bonding wood and wood products are being carried out with the intention to improve their properties, taking into account a lower environmental impact. To this end, new formulations of polyvinyl acetate (PVA) adhesives have been developed, because they have no polluting harmful to health chemicals in their composition, in contrast to formaldehyde-based resins; which in turn are the most commonly used for wood panels production these days. This study tested three different formulations of PVA adhesives, with different pressing times and temperatures for the production of Eucalyptus sp. plywood, obtaining satisfactory results with respect to shear strength at the bondline, which was higher for the PVA adhesives compared to the urea-formaldehyde and phenol-formaldehyde panels found in the literature.
\end{abstract}

Keywords: mechanical properties, Eucalyptus, polyvinyl acetate. 


\section{INTRODUÇÃO}

O setor florestal brasileiro possui grande importância econômica no país, já que o Brasil apresenta uma vasta área de florestas nativas e de reflorestamento. Apesar disso, com o passar dos anos, percebeu-se a necessidade cada vez maior de racionalização da madeira, principalmente através do desenvolvimento de novas tecnologias, que, além de diminuírem a geração de resíduos com a melhor utilização dos recursos florestais, também melhoram algumas de suas propriedades. Com isso começaram a surgir os painéis à base de madeira (compensados, particulados e os painéis de fibras).

Surgiu também a necessidade do desenvolvimento de adesivos que fossem menos agressivos ao meio ambiente, ao passo que novos painéis à base de madeira foram sendo desenvolvidos. Pesquisas estão sendo desenvolvidas neste sentido, procurando diminuir a emissão de poluentes por estes adesivos, mas, ao mesmo tempo, tentando não encarecê-los e, também, não prejudicar as propriedades dos painéis com eles produzidos.

Mamínski et al. (2008) realizaram um estudo no qual foi adicionado glutaraldeído (GA) em proporções de 5 e $10 \%$ ao adesivo ureia-formaldeído (UF), com a intenção de aumentar a resistência à umidade em compensados. Nos compensados de Pinus sylvestris, notou-se um aumento gradual de resistência ao cisalhamento na linha de cola com os painéis produzidos apenas com UF e com 5 e $10 \%$ de GA, tanto em corpos de prova secos quanto nos úmidos (os quais foram submersos em água por 24 horas). Os resultados obtidos para os compensados de faia (Fagus sylvatica) no teste com imersão apresentaram $20 \%$ de aumento no cisalhamento da linha de cola para a proporção de $5 \%$ de GA e um aumento de 53\% para a proporção de $10 \%$ de GA, comparados com os painéis produzidos apenas com UF. Este último apresentou também um valor de cisalhamento na linha de cola no teste com imersão maior que o apresentado para o painel sem imersão produzido apenas com UF, devido ao aumento da resistência à umidade do adesivo e à redução da penetração de água na linha de cola.

Em um estudo realizado por Hoong et al. (2009) utilizando compensado de Kedondong
(Canarium spp.), testou-se a influência da fortificação do adesivo fenol-formaldeído (FF) com tanino sulfitado proveniente da casca de Acacia mangium, e concluiu-se que pode haver uma substituição de até $80 \%$ do FF pelo tanino, o que resultaria em valores de cisalhamento na linha de cola que atendem aos requisitos das normas europeias EN 314-1 e EN 3142:1993.

Moubarik et al. (2009) testaram um adesivo livre de formaldeído composto por maisena e tanino de vime fortificado com hexamina para a colagem de compensados de Pinus pinaster, os resultados apresentaram emissões de formaldeído baixas, que foram provenientes somente da madeira utilizada e não do adesivo, resultando em boas propriedades mecânicas para o compensado, que podem ser comparadas àquelas obtidas para painéis produzidos com fenol-formaldeído.

Despres et al. (2009) também testaram um adesivo livre de formaldeído à base de resina de ureiadimetoxyetanal (Ureia-DME) e um di-isocianato (pMDI) em aglomerados, resultando em painéis de excelentes propriedades com rápido tempo de prensagem e com emissão de formaldeído muito baixa, relativa apenas ao aquecimento da madeira.

Nos exemplos anteriores, nota-se que novas formulações de adesivos vêm sendo desenvolvidas com a intenção de melhorar as propriedades dos painéis e diminuir a agressão ao meio ambiente, causada por adesivos que emitam formaldeído.

Um adesivo, que pode ser utilizado como alternativa aos adesivos convencionais, são os adesivos PVA que, segundo Oliveira \& Dantas (2003), são utilizados em colagens não estruturais de substratos em diversos segmentos da indústria moveleira e/ou madeireira, como, por exemplo: painéis, laminados plásticos e de madeira, colagem de espiga e cavilha, finger-joint, entre outros.

Por serem à base de água, as resinas PVA apresentam vantagens como a facilidade e segurança no manuseio, são inodoras e não inflamáveis, possuem baixo custo se comparadas a outras resinas comerciais, possuem secagem rápida sob condições adequadas, são de fácil limpeza e possuem boa estabilidade à estocagem (IWAKIRI, 2005).

O objetivo deste trabalho foi avaliar a influência de três adesivos à base de PVA com diferentes 
formulações nas propriedades de resistência ao cisalhamento na linha de cola de compensados produzidos com lâminas de Eucalyptus sp., incluindo também a utilização de duas combinações diferentes de tempo e temperatura de prensagem. Bem como comparar os resultados obtidos com os encontrados na literatura para a ureia e o fenol-formaldeído.

\section{MATERIAL E MÉTODOS}

As matérias-primas utilizadas neste trabalho foram lâminas de madeira e três formulações diferentes de adesivos vinílicos, conhecidos como PVA. As lâminas de madeira utilizadas foram da espécie Eucalyptus sp., que foram doadas por uma empresa localizada na cidade de Sengés, Estado do Paraná. Depois do recebimento das lâminas, estas foram esquadrejadas nas dimensões de $50 \times 50 \mathrm{~cm}$, pelo fato da limitação dimensional imposta pela prensa utilizada na prensagem a quente. Este esquadrejamento foi feito de modo a se conseguir um melhor aproveitamento da lâmina, tentando retirar, ao máximo possível, imperfeições como rachaduras, lascas, nós etc. No total foram obtidas 90 lâminas para a produção de 18 painéis de compensado.

Os três adesivos utilizados na produção dos compensados foram nomeados como X, Y e Z, e suas características estão apresentadas a seguir:

- ADESIVO X: é uma dispersão aquosa à base de acetato de polivinila (PVA). Trata-se de um adesivo de alto desempenho, especialmente indicado para a montagem de painéis em prensagem com ou sem aquecimento e radiofrequência e também para finger joint. Possui cor branca, viscosidade entre 2000 e 3000 cP, teor de sólidos de 55-65\% e pH de 2,9-3,5. É um adesivo PVA com classificação D3 de resistência à umidade de acordo com a norma DIN EM-204, o que o qualifica como um adesivo PVA com boa resistência à umidade.
- ADESIVO Y: é uma dispersão aquosa à base de acetato de polivinila (PVA) modificado. Este adesivo é indicado para a colagem de madeiras, trabalhos de bancada ou montagem de painéis, bem como na laminação de HPL (Revestimento de Alta Pressão). Também é indicado para prensas a quente e para colagem de madeira com concreto (colagem de parquet) e nivelamento de pisos. Possui cor branca, viscosidade entre 9500 e 14000 cP, teor de sólidos de 55-60\% e pH de $3,5-5,5$.

- ADESIVO Z: é uma dispersão aquosa de acetato de polivinila com plastificação externa. Este adesivo é indicado para a colagem de madeiras, trabalhos de bancada ou montagem de painéis, bem como na laminação de HPL (Revestimento de Alta Pressão). Também é indicado para prensas a quente. Possui cor branca, viscosidade entre 8000 e $12000 \mathrm{cP}$, teor de sólidos de 48-52\% e pH de 4,0-6,0.

\subsection{Produção dos painéis}

Foram definidas seis condições de produção levando-se em consideração o adesivo utilizado, o tempo e a temperatura de prensagem. A Tabela 1 apresenta as características das seis condições de produção e a nomenclatura de cada painel produzido.

Foram produzidos, no total, 18 painéis, sendo três para cada condição de produção. A gramatura utilizada para os três adesivos foi de 120 g.m ${ }^{-2}$ para linha simples, a recomendada pelo fabricante dos adesivos. Como o painel possuía dimensões aproximadas de $50 \times 50 \mathrm{~cm}$, para cada uma das quatro linhas de cola do painel deveriam ser pesadas $30 \mathrm{~g}$ de adesivo (valor este obtido através da gramatura estipulada e da área da linha de cola). Deste modo, foram pesadas as quantidades de cola necessárias para cada linha de cola dos painéis, ou seja, quatro porções por painel, com $10 \mathrm{~g}$ a mais do que o necessário, sendo então $40 \mathrm{~g}$, pois se notou que

Tabela 1. Composição das seis condições de produção estudadas.

Table 1. Composition of the six production conditions studied.

\begin{tabular}{cccc} 
Condições & Adesivo & Tempo de prensagem [min] & Temperatura de prensagem $\left[{ }^{\circ} \mathrm{C}\right]$ \\
\hline 1 & $\mathrm{X}$ & 15 & 60 \\
2 & $\mathrm{X}$ & 10 & 90 \\
\hline 3 & $\mathrm{Y}$ & 15 & 60 \\
\hline 4 & $\mathrm{Y}$ & 10 & 90 \\
\hline 5 & $\mathrm{Z}$ & 15 & 60 \\
\hline 6 & $\mathrm{Z}$ & 10 & 90 \\
\hline
\end{tabular}


uma parte do adesivo ficaria retida no recipiente. E então houve a aplicação dos adesivos nas lâminas e a montagem do painel.

Posteriormente, ocorreu a prensagem do painel, que foi realizada em uma prensa hidráulica termomecânica da marca Hidral-Mac com capacidade de carga de 80 kgf e temperatura máxima de $200{ }^{\circ} \mathrm{C}$. Primeiramente, foram prensados todos os painéis com temperatura de $60{ }^{\circ} \mathrm{C}$ e tempo de prensagem de 15 minutos e, posteriormente, os painéis com $90{ }^{\circ} \mathrm{C}$ e tempo de prensagem de 10 minutos. Durante a prensagem, foi mantida a pressão constante de 15 BAR (15,3 kgf. $\left.\mathrm{cm}^{-2}\right)$.

\subsection{Ensaio de cisalhamento na linha de cola}

Os ensaios de cisalhamento na linha de cola foram realizados de acordo com a norma da ABNT (1986) NBR ISO 12466-1. Primeiramente, foram confeccionados seis corpos de prova por tratamento, sendo dois de cada um dos três painéis constituintes das condições. As dimensões das amostras estão apresentadas na Figura 1, na qual se percebe que os rasgos de ambos os lados alcançam o centro da terceira lâmina ou lâmina central do painel. As lâminas externas e central possuem a direção das fibras paralela ao comprimento dos corpos de prova, com a finalidade de que as outras duas lâminas, entre as linhas de colas testadas, estejam com a direção das fibras perpendicular ao comprimento, de acordo com a norma.

Os painéis por serem produzidos com adesivo vinílico, não apresentam característica de resistência à umidade suficiente, por este motivo os corpos de prova não passaram por um pré-tratamento. Os ensaios foram realizados na máquina universal de

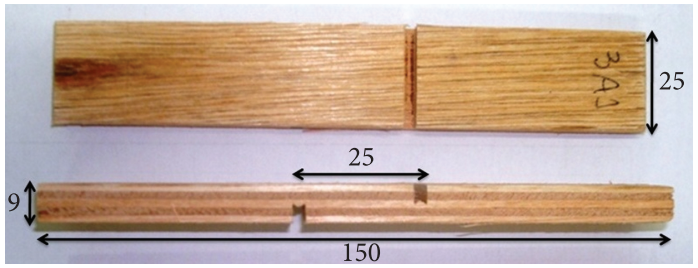

Figura 1. Corpo de prova de cisalhamento na linha de cola (dimensões em mm).

Figure 1. Shear strength at the bondline specimen (dimensions in $\mathrm{mm}$ ). ensaios EMIC do Laboratório de Propriedade dos Materiais - na UNESP - Campus de Itapeva, na qual os corpos de prova foram ensaiados com velocidade de $120 \mathrm{~N} . \mathrm{s}^{-1}$, proporcionando um tempo de ensaio médio de 35 segundos, dentro do exigido pela norma que é de $30 \pm 10$ segundos. A Figura 2 apresenta o corpo de prova sendo ensaiado.

\subsection{Análise estatística}

Foi realizado um delineamento fatorial com dois fatores, sendo um correspondente aos adesivos e outro correspondente à combinação T/t. O fator correspondente aos adesivos possui três níveis, sendo cada nível um dos adesivos utilizados, e o fator correspondente à combinação $\mathrm{T} / \mathrm{t}$ possui dois níveis, sendo um de $60{ }^{\circ} \mathrm{C}$ e 15 minutos e o outro de $90{ }^{\circ} \mathrm{C}$ e 10 minutos, resultando em seis níveis que correspondem às seis condições de produção descritas na seção 4.1 .

A análise estatística utilizada foi a Análise de Variância com nível de significância de 5\% para testar a existência de diferenças significativas entre as médias. Nos casos em que houve diferenças significativas foi necessário realizar o Teste de Tukey, com o intuito de identificar estas diferenças. Para a análise estatística foi utilizado o software R Development versão 2.10.0 (2009).

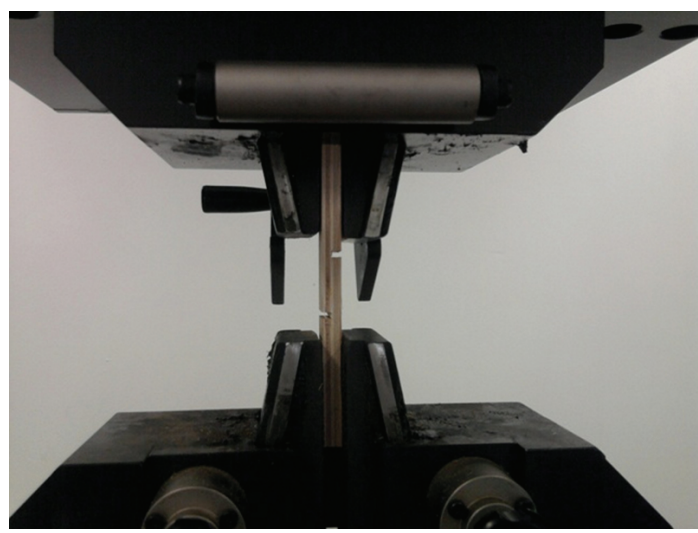

Figura 2. Corpo de prova de cisalhamento na linha de cola na máquina de ensaios.

Figure 2. Shear strength at the bondline specimen in the testing machine. 
Tabela 2. Médias do cisalhamento na linha de cola (MPa) para cada condição de produção.

Table 2. Shear strength at the bondline averages (MPa) for each condition of production.

\begin{tabular}{|c|c|c|c|c|}
\hline & $\mathbf{X}$ & $\mathbf{Y}$ & $\mathbf{Z}$ & Média T/t \\
\hline $60^{\circ} \mathrm{C} / 15 \mathrm{~min}$. & 2,61 & 2,97 & 3,29 & $2,96^{\mathrm{a}}$ \\
\hline $90^{\circ} \mathrm{C} / 10 \mathrm{~min}$. & 3,58 & 3,44 & 3,16 & $3,39^{\mathrm{a}}$ \\
\hline Média adesivos & $3,09^{A \star}$ & $3,21^{\mathrm{A}}$ & $3,22^{\mathrm{A}}$ & \\
\hline
\end{tabular}

\section{RESULTADOS E DISCUSSÃO}

A Tabela 2 apresenta os resultados médios de cisalhamento na linha de cola obtidos para todas as condições de produção.

A análise de variância realizada para os adesivos $\left(\mathrm{F}_{2,30}=0,06 ; \mathrm{p}>0,05\right)$ e para as combinações $\mathrm{T} / \mathrm{t}$ $\left(\mathrm{F}_{1,30}=1,78 ; \mathrm{p}>0,05\right)$ mostrou que não houve diferenças significativas, e para as seis condições de produção $\left(\mathrm{F}_{2,30}=0,95 ; \mathrm{p}>0,05\right)$ não ocorreu interação. Esta é uma tendência diferente do que ocorreu na flexão estática, na qual o adesivo PVA 1810 possuiu valores superiores aos outros. Nota-se que, para o cisalhamento na linha de cola, os três adesivos possuem as mesmas características, não diferindo entre si, e também não houve influência do tempo ou da temperatura. A Figura 3 apresenta o gráfico com as médias dos valores em cada condição de produção.

No cisalhamento da linha de cola, houve também uma tendência diferente se comparado com outros adesivos.

No estudo realizado por Bortoletto Júnior (2003) com fenol-formaldeído, esta característica variou de 2,5 a 2,7 $\mathrm{MPa}$, enquanto as médias para os três adesivos PVA foram superiores a $3 \mathrm{MPa}$, ou seja, comparando-se com o FF que é um adesivo estrutural, os adesivos PVA testados apresentaram valores superiores.

Almeida et al. (2004) obtiveram valores de 1,91 e 1,68 MPa, ou seja, os adesivos PVA mostraramse superiores se comparados também com a ureiaformaldeído.

Um estudo realizado por Çolakoglu \& Demirkir (2005), utilizando-se ureia-formaldeído com bórax em compensados, mostrou também resultados equivalentes à metade dos obtidos com os três adesivos PVA.

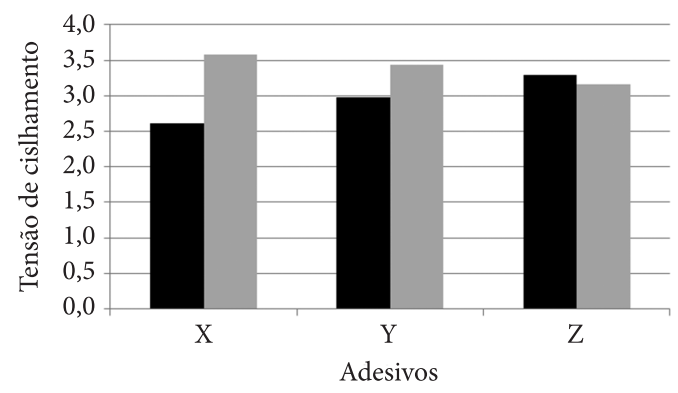

- $60{ }^{\circ} \mathrm{C} / 15 \mathrm{~min} \quad 90^{\circ} \mathrm{C} / 10 \mathrm{~min}$

Figura 3. Resistência ao cisalhamento na linha de cola para as diferentes condições de prensagem e formulações empregadas.

Figure 3. Shear strength at the bondline to the different pressing conditions and formulations employed.

Portanto, no caso do cisalhamento na linha de cola, os adesivos testados mostraram-se superiores tanto em relação ao FF quanto à UF, podendo substituir estes adesivos em condições nas quais o cisalhamento na linha de cola torna-se a característica mais exigida.

\section{CONCLUSÕES}

Os três adesivos apresentaram valores adequados de resistência ao cisalhamento na linha cola. Tal resultado pode ser justificado pelo fato do adesivo PVA ter como característica seu bom desempenho plástico, o que foi favorável quando solicitado ao esforço de cisalhamento, pois ele absorveu a deformação plástica e conseguiu aumentar a resistência nesta solicitação.

Não houve influência das combinações tempo e temperatura de prensagem, já que os resultados obtidos não apresentaram diferenças estatisticamente significativas.

Os resultados obtidos para os compensados com PVA, quando comparados aos da literatura, 
para compensados de ureia ou fenol-formaldeído, revelaram resultados de resistência ao cisalhamento superiores, portanto, para esforços de cisalhamento, os adesivos PVA podem ser bons substitutos aos adesivos convencionais.

\section{STATUS DA SUBMISSÃO}

Recebido: 16/09/2011

Aceito: 07/02/2012

Resumo publicado online: 22/03/2012

Artigo completo publicado: 30/06/2012

\section{AUTOR(ES) PARA CORRESPONDÊNCIA}

\section{Cristiane Inácio de Campos}

Universidade Estadual Paulista "Júlio de

Mesquita Filho" - UNESP,

Campus Experimental de Itapeva,

Rua Geraldo Alckmin, 519,

Vila Nossa Senhora de Fátima,

CEP 18409-010, Itapeva, SP, Brasil.

e-mail: cristiane@itapeva.unesp.br

\section{REFERÊNCIAS}

Associação Brasileira de Normas Técnicas - ABNT. NBR ISO 12466-1: Madeira Compensada - Qualidade de colagem parte 1: métodos de ensaio. Rio de Janeiro: ABNT; 1986.

Almeida RR, Bortoletto Júnior G, Jamkowsky IP. Produção de compensados a partir da madeira de clones do híbrido Eucalyptus grandis x Eucalyptus urophylla. Floresta e Ambiente 2004; 11(1): 14-24.

Bortoletto Júnior G. Produção de compensados com 11 espécies do gênero Eucalyptus, avaliação das suas propriedades físico-mecânicas e indicações para utilização. Scientia Forestalis 2003; (63): 65-78.

Çolakoglu G, Demirkir C. Characteristics of plywood panels produced with urea formaldehyde resin (UF) containing borax. European Journal of Wood and Wood Products 2005; 64(3): 250-251. http://dx.doi. org/10.1007/s00107-005-0077-5

Despres A, Pizzi A, Vu C, Delmotte L. Colourless formaldehyde-free urea resin adhesives for wood panels. European Journal of Wood and Wood Products 2009; 68(1): 13-20. http://dx.doi.org/10.1007/s00107009-0344-y

Hoong YB, Paridah MT, Luqman CA, Koh MP, Loh YF. Fortification of sulfited tannin from the bark of Acacia mangium with phenol-formaldehyde for use as plywood adhesive. Industrial Crops and Products 2009; 30(3): 416421. http://dx.doi.org/10.1016/j.indcrop.2009.07.012

Maminski ML, Borysiuk P, Zado A. Study on the water resistance of plywood bonded with UF-glutaraldehyde adhesive. European Journal of Wood and Wood Products 2008; 66(6): 469-470. http://dx.doi.org/10.1007/s00107008-0244-6

Iwakiri, S. Painéis de madeira reconstituída. Curtiba: FUPEF, 2005. 247 p.

Moubarik A, Allal A, Pizzi A, Charrier F, Charrier B. Characterization of a formaldehyde-free cornstarchtannin wood adhesive for interior plywood. European Journal of Wood and Wood Products 2009; 68(4): 427433. http://dx.doi.org/10.1007/s00107-009-0379-0

Oliveira, M. P.; Dantas, W. C. V. Evolução dos adesivos ecologicamente corretos para o setor madeireiro. In: II Seminário de Produtos Sólidos de Madeira de Eucalipto - SIF. Revista da madeira, set. 2003.

R Development Core Team. R: A language and environment for statistical computing. version 2.10.0. Vienna: R Foundation for Statistical Computing; 2009. [cited 2011 jul. 10]. Available from: http://www.Rproject.org. 